\title{
Increasing the accuracy of evaluation in selecting a location for establishing an intermodal terminal
}

\author{
Svetoslav Martinov ${ }^{1, *}$ \\ ${ }^{1}$ Technical University - Sofia, Department of Railway Engineering, Bulgaria
}

\begin{abstract}
The present study is aimed at increasing the accuracy of multi-criteria evaluation of alternatives in selecting a location for establishing an intermodal terminal in the North-Central planning development region of Bulgaria. A model has been used in the paper that allows us to increase the accuracy of multicriteria evaluation of all studied alternatives. This has been achieved by converting the values of various units into identical dimensionless units within a defined interval. It makes it possible to do an evaluation of the alternatives by using the real values of the criteria. The model has been used for multi-criteria evaluation of the alternatives in selecting a location for establishing an intermodal terminal in the city of Ruse. The results have been compared with the results of a pre-feasibility study of establishing an intermodal terminal in the city of Ruse in the North-Central planning development region of Bulgaria.
\end{abstract}

\section{Introduction}

Some of the guidelines of the Integrated Transport Strategy for the period of up to 2030 are related to the development of the transport network in Bulgaria as part of the Trans-European Transport Network (TEN-T) and to increasing the efficiency and competitiveness of the transport sector [1]. The investment strategy of the Operational program "Transport and Transport Infrastructure" 2014-2020, under priority axe 3 referring to intermodal transport, envisages the development of a network of intermodal terminals for improving intermodality [2] of freight transport. Part of the investment strategy is Project "Construction of an intermodal terminal in the North-Central planning development region in Bulgaria - Ruse". The preparatory activities for the establishment of the terminal are carried out under Project "Technical assistance for construction of intermodal terminal in North-Central planning development region in Bulgaria - Ruse" [3], including the selection of a terminal location. Five alternative locations for establishing the intermodal terminal have been discussed [3]. The Weighted Sum Method (WSM) has been applied in the project [3] to evaluate different alternatives and to select a leading alternative.

The Weighted Sum Method is one of the most commonly used multi-criteria decision making methods $[4,5]$, especially in single dimensional type problems [6]. The choice of a decision among many alternatives often depends on different parameters. This requires the use of multi-criteria analysis methods [6-9]. The weight multicriteria analysis methods are used in different fields of transport, including for selection of a location of terminal $[9,10]$. The evaluation of the alternatives in [3] has been carried out through different criteria that are estimated using a three-tier rating scale [3] and weight coefficients. The three-tier rating scale allows for alternatives with different values of the criteria to be put in the same group. That could lead to an inaccurate final rating of the alternatives. For alternatives evaluated through a lot of criteria $[11,12]$, the accumulated inaccuracies could lead to a significant error and, consequently, to an incorrect final ranking.

\section{Methodology of research}

\subsection{Model for evaluation and selection of an alternative ("As-Is" scenario)}

Five possible alternatives for the construction of the terminal have been studied in project [3]. In the paper we will call this scenario "As-Is" scenario. In the project, the alternative places for the establishment of the terminal are represented by designations A, B, C, D and E [3].

The Weighted Sum Method has been used in selecting a location for establishing an intermodal terminal - Ruse [3]. The method is based on a comparison between different alternatives by determining their weighted sums. The weighted sum for each alternative is calculated through the value of the alternative and its individual importance (weight coefficient) [5]. The Weighted Sum Method allows to compare variants with the same dimensions of the criteria [13].

The project [3] describes all the criteria and their relative weight. Criteria (C) are divided into five main groups that contain relevant sub-criteria (SC) [3]: Criterion 1 - Impact on existing infrastructure and the environment; Sub-criterion 1.1 - Strategic location of the site; Sub-criterion 1.2 - Purpose of the land; Sub-criterion

\footnotetext{
* Corresponding author: s.martinov@tu-sofia.bg
} 
1.3 - Land ownership; Sub-criterion 1.4 - Environment and European protected areas - Natura 2000; Subcriterion 1.5 - Effect on communal infrastructure; Criterion 2 - Opportunities for development of a place for intermodal terminal; Sub-criterion 2.1 - Size of the site; Sub-criterion 2.2 - Length of the longest arrival-departure railway track; Sub-criterion 2.3 - Average useful length of railway tracks used for loading/unloading; Subcriterion 2.4 - Opportunity for further development; Criterion 3 - Transport access to the intermodal terminal; Sub-criterion 3.1 - Railway infrastructure; Sub-criterion 3.2 - Road infrastructure; Sub-criterion 3.3 - Port infrastructure; Criterion 4 - Costs and operating parameters of the intermodal terminal; Sub-criterion $4.1-$ Investment costs; Sub-criterion 4.2 - Annual operating and maintenance costs; Sub-criterion 4.3 - Opportunities for development of additional services; Sub-criterion 4.4 - Quantity of manipulated Twenty-foot equivalent units (TEU); Criterion 5- Economical parameters of the intermodal terminal; Sub-criterion 5.1 - Net present value (NPV) of the total costs; Sub-criterion 5.2 - Sensitivity of NPV to operating and maintenance costs; Sub-criterion 5.3 - NPV ratio of the total costs per TEU.

The estimates of the sub-criteria of the different alternatives and their weight coefficients are shown in Table 1. The type of criteria and sub-criteria and their weights are proposed in the documentation of [3]. The sub-criteria in the project are evaluated by a three-tier scale in which the preferred level is rated as 1 , the average level is rated as 2 and the critical level is rated as 3 [3].

Table 1. Estimates and weight coefficients.

\begin{tabular}{|l|c|c|c|c|c|c|}
\hline Criterion (C)/ & Weight & \multicolumn{5}{|c|}{ Alternatives } \\
\cline { 4 - 7 } Sub-criterion (SC) & coefficient & A & B & C & D & E \\
\hline C 1 & 0,15 & - & - & - & - & - \\
\hline SC 1.1 & 0,2 & 3 & 3 & 1 & 3 & 3 \\
\hline SC 1.2 & 0,3 & 1 & 2 & 1 & 1 & 3 \\
\hline SC 1.3 & 0,2 & 2 & 2 & 1 & 2 & 2 \\
\hline SC 1.4 & 0,2 & 1 & 1 & 1 & 1 & 2 \\
\hline SC 1.5 & 0,1 & 2 & 2 & 1 & 2 & 1 \\
\hline C 2 & 0,2 & - & - & - & - & - \\
\hline SC 2.1 & 0,25 & 2 & 2 & 1 & 2 & 2 \\
\hline SC 2.2 & 0,25 & 2 & 2 & 1 & 3 & 2 \\
\hline SC 2.3 & 0,25 & 2 & 1 & 1 & 2 & 1 \\
\hline SC 2.4 & 0,25 & 3 & 1 & 1 & 3 & 2 \\
\hline C 3 & 0,2 & - & - & - & - & - \\
\hline SC 3.1 & 0,4 & 1 & 2 & 1 & 2 & 2 \\
\hline SC 3.2 & 0,35 & 3 & 1 & 1 & 2 & 3 \\
\hline SC 3.3 & 0,25 & 2 & 2 & 2 & 1 & 1 \\
\hline C 4 & 0,2 & - & - & - & - & - \\
\hline SC 4.1 & 0,25 & 1 & 2 & 1 & 2 & 3 \\
\hline SC 4.2 & 0,25 & 1 & 2 & 2 & 2 & 3 \\
\hline SC 4.3 & 0,25 & 1 & 1 & 1 & 1 & 1 \\
\hline SC 4.4 & 0,25 & 2 & 2 & 1 & 3 & 2 \\
\hline C 5 & 0,25 & - & - & - & - & - \\
\hline SC 5.1 & 0,3 & 1 & 2 & 2 & 2 & 3 \\
\hline SC 5.2 & 0,3 & 1 & 2 & 1 & 3 & 3 \\
\hline SC 5.3 & 0,4 & 1 & 2 & 1 & 3 & 3 \\
\hline
\end{tabular}

The project [3] does not contain information or data about the matrix of pairwise comparisons [7, 14] of the criteria and the sub-criteria. The weights of the criteria and the sub-criteria are described in project documentation and their values [3] are used in the paper. For that reason a sensitivity analysis of weights coefficients and values of the consistency ration $[7,14]$ is not presented in the research.

Evaluation of the criteria through the sub-criteria and of the alternatives through the criteria in the project [3] is carried out by:

$$
a_{n r}=\sum_{s=1}^{S} a_{s r} w_{s}, n=1,2, \ldots, N ; r=1,2, \ldots, R
$$

and

$$
A_{r}=\sum_{n=1}^{N} a_{n r} w_{n}, r=1,2, \ldots, R,
$$

where $a_{n r}$ is the estimate of the n-th decision criterion for the $r$-th alternative; $N$ - the number of the same type criteria; $R$ - the number of the compared alternatives $(R \geq 2) ; a_{s r}-$ the estimate of the s-th decision subcriterion for the r-th alternative; $w_{S}$ - weight coefficient of the s-th sub-criterion $\left(\sum_{s=1}^{S} w_{S}=1\right) ; S$ - the number of the same type sub-criteria; $A_{r}$ - the value of the weighted sum for the r-th alternative; $w_{n}$ - weight coefficient of the $n$-th criterion $\left(\sum_{n=1}^{N} w_{n}=1\right)$.

The selection of a leading alternative is made by [3]:

$$
A_{r} \rightarrow \min , r=1,2, \ldots, R \text {. }
$$

\subsection{Model for increasing the accuracy of estimates ("To-Be" scenario)}

For the purpose of increasing the accuracy of multicriteria evaluation of the alternatives in selecting a location for construction of an intermodal terminal in the city of Ruse the values of the parameters are converted into dimensionless units. The parameters of the criteria that have a numerical value and a corresponding dimension are converted into dimensionless units within a defined interval [15]. In the paper we will call this scenario "To-Be" scenario. The conversion for $r=1,2, \ldots, R$ is done through equation (4) for the direct ratio criteria and through equation (5) for the inverse ratio criteria [16]:

$$
\begin{aligned}
& M_{s r}=L_{s r, \text { max }} \cdot \frac{\left(V_{s r}-V_{s, \text { min }}\right)}{\left(V_{s, \text { max }}-V_{s, \text { min }}\right)}+L_{s r, \text { min }} \\
& M_{s r}=L_{s r, \text { max }} \cdot \frac{\left(V_{s, \text { max }}-V_{s r}\right)}{\left(V_{s, \text { max }}-V_{s, \text { min }}\right)}+L_{s r, \text { min }},
\end{aligned}
$$

where $M_{s r}$ is the dimensionless estimate of the $s$-th decision sub-criterion $(s=1,2, \ldots, S)$ for the $r$-th alternative; $L_{s r, \min }$ and $L_{s r, \text { max }}$ - respectively the minimum and the maximum values determining the length of the interval of conversion of the estimate of the $s$-th sub-criterion of the $r$-th alternative; $V_{s r}$ - the current value of the s-th sub-criterion of the r-th alternative; $V_{s, \min }$ - the minimum possible value of the $s$-th sub-criterion; $V_{s, \text { max }}$ - the maximum possible value of the $s$-th subcriterion.

The values of $L_{s r, \min }$ and $L_{s r, \max }$ are:

$$
\begin{aligned}
& L_{s r, \text { min }} \geq 0 ; \\
& L_{s r, \text { max }}>0 .
\end{aligned}
$$


The interval of limit [16] of estimate $M_{s r}$ is determined by the values of $L_{s r, \min }$ and $L_{s r, \max }$ :

$$
M_{s r} \in\left[L_{s r, \min }, L_{s r, \min }+L_{s r, \max }\right] .
$$

When converted into dimensionless estimates the values of $V_{s, \min }$ and $V_{s, \max }$ for $s=1,2, \ldots, S$ could be determined [16] using:

$$
\begin{aligned}
& V_{s, \text { min }}=\min V_{s r}, r=1,2, \ldots, R ; \\
& V_{s, \text { max }}=\max V_{s r}, r=1,2, \ldots, R .
\end{aligned}
$$

The limits [16] for the value of $V_{s, \min }$ and $V_{s, \max }$ are:

$$
0 \leq V_{s, \min }<V_{s, \max }
$$

When it is not necessary for some parameters to be converted into dimensionless units, the value of $M_{s r}=V_{s r}$ for $s=1,2, \ldots, S$ and $r=1,2, \ldots, R$ or it could be calculated with equations (4) and (5) for $V_{s, \min }=L_{s r, \min }$ and for $V_{s, \text { max }}=L_{s r, \text { min }}+L_{s r, \text { max }}$.

The evaluation of the criteria through the sub-criteria and of the alternatives through the criteria for the "To-Be" scenario is carried out for $r=1,2, \ldots, R$ through:

$$
a_{n r}^{c}=\sum_{s=1}^{S} M_{s r} w_{s}, n=1,2, \ldots, N
$$

and

$$
A_{r}^{c}=\sum_{n=1}^{N} a_{n r}^{c} w_{n}
$$

where $a_{n r}^{c}$ is the estimate with a higher accuracy of the $n$-th decision criterion for the $r$-th alternative; $A_{r}^{c}-$ the value of the weighted sum for the $r$-th alternative after applying the model for increasing the accuracy [16].

The choice of a leading alternative is carried out with:

$$
A_{r}^{c} \rightarrow \min , r=1,2, \ldots, R .
$$

\section{Results and discussion}

\section{1 “As-Is" scenario}

The estimates of the criteria and alternatives for the "AsIs" scenario are shown in Table 2. A graphical comparison between the estimates of the alternatives is shown in Fig. 1.

Table 2. Estimates of the criteria and alternatives

\begin{tabular}{|c|c|c|c|c|c|}
\hline \multirow{2}{*}{ Criterion } & \multicolumn{5}{|c|}{ Alternatives } \\
\cline { 2 - 6 } & $\mathbf{A}$ & $\mathbf{B}$ & $\mathbf{C}$ & $\mathbf{D}$ & $\mathbf{E}$ \\
\hline Criterion 1 & 1,70 & 2,00 & 1,00 & 1,70 & 2,40 \\
\hline Criterion 2 & 2,25 & 1,50 & 1,00 & 2,50 & 1,75 \\
\hline Criterion 3 & 1,95 & 1,65 & 1,25 & 1,75 & 2,10 \\
\hline Criterion 4 & 1,25 & 1,75 & 1,25 & 2,00 & 2,25 \\
\hline Criterion 5 & 1,00 & 2,00 & 1,30 & 2,70 & 3,00 \\
\hline $\begin{array}{c}\text { Estimate of } \\
\text { alternative }\end{array}$ & 1,60 & 1,78 & 1,18 & 2,18 & 2,33 \\
\hline
\end{tabular}

As we could see from the results, the leading alternative in the project [3] is alternative $\mathrm{C}$, followed by alternatives A, B, D and E.

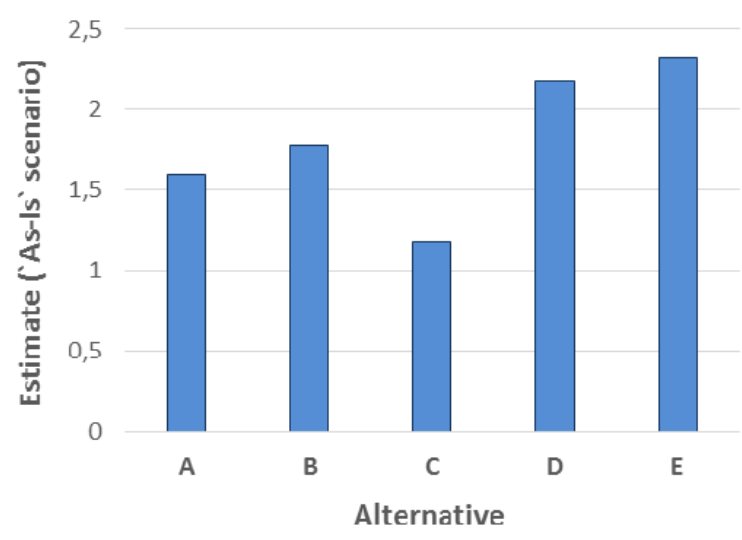

Fig. 1. Comparison of the alternatives.

\section{2 "To-Be" scenario}

The model for conversion of the values of the parameters into dimensionless estimates [16] is applied to increase the accuracy of the multi-criteria evaluation of the alternatives in choosing a location for the construction of an intermodal terminal in the North-Central planning development region in Bulgaria - Ruse.

The selection of a location of the terminal has been made by applying nineteen sub-criteria that are shown in Table 1. In the project documentation [3], all sub-criteria have been evaluated by using the three-tier scale with estimates of 1, 2 or 3. Nine of the sub-criteria (SC 2.1, SC 2.2, SC 2.3, SC 3.2, SC 3.3, SC 4.1, SC 4.2, SC 4.4 and $\mathrm{SC} 5.1)$ are parameters with the respective numerical value and dimension [3] shown in Table 3.

Table 3. Values of the sub-criteria.

\begin{tabular}{|l|c|c|c|c|c|}
\hline \multirow{2}{*}{ Sub-criteria, dimension } & \multicolumn{5}{|c|}{ Alternatives } \\
\cline { 2 - 6 } & A & B & C & D & E \\
\hline SC 2.1, 1000 m ${ }^{2}$ & 109,3 & 109,8 & 123,4 & 96,0 & 112,1 \\
\hline SC 2.2, m & 760 & 750 & 774 & 636 & 750 \\
\hline SC 2.3, m & 470 & 530 & 530 & 470 & 530 \\
\hline SC 3.2, m & 1050 & 220 & 100 & 580 & 850 \\
\hline SC 3.3, m & 5800 & 3500 & 5400 & 500 & 400 \\
\hline SC 4.1, million EUR & 21,7 & 22,2 & 21,8 & 24,8 & 27,9 \\
\hline SC 4.2, 1000 EUR & 399,0 & 419,4 & 423,7 & 468,2 & 554,2 \\
\hline SC 4.4, 1000 TEU & 30,6 & 30,7 & 34,6 & 26,9 & 31,4 \\
\hline SC 5.1, million EUR & 29,1 & 29,8 & 29,6 & 33,1 & 37,3 \\
\hline
\end{tabular}

The choice of an optimal value, minimum or maximum for the target function, is being made depending on the type of target function - costs or benefits and the type of the evaluated criterion - proportionate (in direct proportion) or disproportionate (in inverse proportion) [16]. Table 4 shows the type (proportionate or disproportionate) of the used sub-criteria with a numerical value and dimension SC 2.1, SC 2.2, SC 2.3, SC 3.2, SC 3.3, SC 4.1, SC 4.2, SC 4.4 and SC 5.1 for which the accuracy of evaluation is increased.

The choice of a correlation to determine the value of the $M_{s r}$ estimate is made according to the type of target function - costs or benefits and the optimal value for choosing an option - minimum or maximum. Table 5 shows a matrix with combinations for choosing a correlation (proportionate or disproportionate) to 
determine the value of estimate $M_{s r}$ depending on the target function (costs or benefits) and the optimal value (minimum or maximum) of the target function. As can be seen in Table 5, with a minimum (min) value for choosing an option and a target function of the costs or benefit type we have to use proportionate (in direct ratio) and disproportionate (in inverse ratio) correlation for estimating the value of $M_{s r}$. On the contrary, with a maximum (max) value for choosing an option and a target of the costs or benefits type, a disproportionate (inverse ratio) or proportionate (direct ratio) correlation is used.

Table 4. Type of sub-criteria.

\begin{tabular}{|c|c|}
\hline Sub-criterion & Type of sub-criterion \\
\hline SC 2.1 & inverse ratio \\
\hline SC 2.2 & inverse ratio \\
\hline SC 2.3 & inverse ratio \\
\hline SC 3.2 & direct ratio \\
\hline SC 3.3 & direct ratio \\
\hline SC 4.1 & direct ratio \\
\hline SC 4.2 & direct ratio \\
\hline SC 4.4 & inverse ratio \\
\hline SC 5.1 & direct ratio \\
\hline
\end{tabular}

Table 5. Matrix of combinations.

\begin{tabular}{|c|c|c|}
\hline Target function & Choice of an option & Type of criteria \\
\hline \multirow{2}{*}{ Cost } & $\min$ & direct ratio \\
\cline { 2 - 3 } & $\max$ & inverse ratio \\
\hline \multirow{2}{*}{ Benefit } & $\max$ & direct ratio \\
\cline { 2 - 3 } & $\min$ & inverse ratio \\
\hline
\end{tabular}

Table 6 shows the values of sub-criteria SC 2.1, SC 2.2, SC 2.3, SC 3.2, SC 3.3, SC 4.1, SC 4.2, SC 4.4 and SC 5.1 by alternatives after their transformation into dimensionless estimate $M_{s r}$. The transformation is made by using the model described in point 2.2. The change of the estimates for the "To-Be" scenario is within a range with a lower limit 1 and upper limit 3 . The range of the change of the interval coincides with the minimum and maximum estimate of the criteria in the project [3].

Table 6. Values of $M_{s r}$.

\begin{tabular}{|c|c|c|c|c|c|}
\hline \multirow{2}{*}{ Sub-criterion } & \multicolumn{5}{|c|}{$\boldsymbol{M}_{\boldsymbol{s} \boldsymbol{r}}$} \\
\cline { 2 - 6 } & \multicolumn{5}{|c|}{ Alternatives } \\
\cline { 2 - 6 } & $\mathbf{A}$ & $\mathbf{B}$ & $\mathbf{C}$ & $\mathbf{D}$ & $\mathbf{E}$ \\
\hline SC 2.1 & 2,03 & 1,99 & 1,00 & 3,00 & 1,82 \\
\hline SC 2.2 & 1,20 & 1,35 & 1,00 & 3,00 & 1,35 \\
\hline SC 2.3 & 3,00 & 1,00 & 1,00 & 3,00 & 1,00 \\
\hline SC 3.2 & 3,00 & 1,25 & 1,00 & 2,01 & 2,58 \\
\hline SC 3.3 & 3,00 & 2,15 & 2,85 & 1,04 & 1,00 \\
\hline SC 4.1 & 1,00 & 1,16 & 1,03 & 2,01 & 3,00 \\
\hline SC 4.2 & 1,00 & 1,26 & 1,32 & 1,89 & 3,00 \\
\hline SC 4.4 & 2,03 & 1,99 & 1,00 & 3,00 & 1,82 \\
\hline SC 5.1 & 1,00 & 1,16 & 1,11 & 1,98 & 3,00 \\
\hline
\end{tabular}

The estimates of the criteria and the results of the multi-criteria evaluation of the alternatives after increasing the accuracy of the calculations are shown in Table 7.

A comparison between the estimates of the alternatives after increasing their accuracy is shown in Fig. 2.
Table 7. Estimates of the criteria and alternatives.

\begin{tabular}{|c|c|c|c|c|c|}
\hline \multirow{2}{*}{ Criterion } & \multicolumn{5}{|c|}{ Alternatives } \\
\cline { 2 - 6 } & $\mathbf{A}$ & $\mathbf{B}$ & $\mathbf{C}$ & $\mathbf{D}$ & $\mathbf{E}$ \\
\hline Criterion 1 & 1,70 & 2,00 & 1,00 & 1,70 & 2,40 \\
\hline Criterion 2 & 2,31 & 1,34 & 1,00 & 3,00 & 1,54 \\
\hline Criterion 3 & 2,20 & 1,78 & 1,46 & 1,76 & 1,95 \\
\hline Criterion 4 & 1,26 & 1,35 & 1,09 & 1,98 & 2,21 \\
\hline Criterion 5 & 1,00 & 1,75 & 1,03 & 2,69 & 3,00 \\
\hline $\begin{array}{c}\text { Estimate of } \\
\text { alternative }\end{array}$ & 1,66 & 1,63 & 1,12 & 2,28 & 2,25 \\
\hline
\end{tabular}

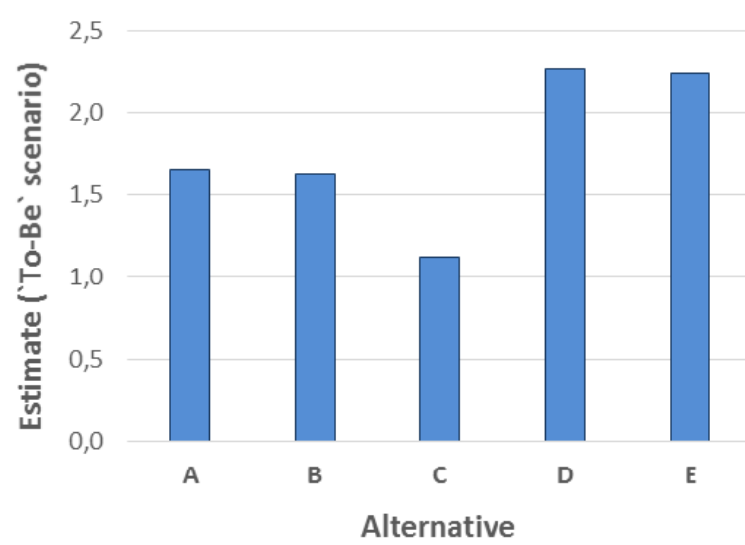

Fig. 2. Comparison of the alternatives.

The estimates in "To-Be" scenario are determined by using the same criteria, sub-criteria and weight coefficients as in "As-Is" scenario. The weights of the criteria are determined in [3]. The evaluation of the alternatives is made by using the same weight coefficients in both "As-Is" and "To-Be" scenarios.

A comparison of the rating of the alternatives before and after increasing the accuracy of the estimates is shown in Table 8.

Table 8. Comparison matrix.

\begin{tabular}{|c|c|c|c|c|c|c|}
\hline \multirow{2}{*}{ Scenario } & \multicolumn{5}{|c|}{ Rating of the alternatives } \\
\cline { 3 - 7 } & $1^{\text {st }}$ & $2^{\text {nd }}$ & $3^{\text {rd }}$ & $4^{\text {th }}$ & $5^{\text {th }}$ \\
\hline \multirow{2}{*}{ "As-Is" } & Alternative & C & A & B & D & E \\
\cline { 2 - 7 } & Estimate & 1,18 & 1,60 & 1,78 & 2,18 & 2,33 \\
\hline \multirow{2}{*}{ "To-Be" } & Alternative & $\mathrm{C}$ & $\mathrm{B}$ & $\mathrm{A}$ & $\mathrm{E}$ & $\mathrm{D}$ \\
\cline { 2 - 7 } & Estimate & 1,12 & 1,63 & 1,66 & 2,25 & 2,28 \\
\hline
\end{tabular}

The comparison of the results (Table 8) from the evaluation of the alternatives for both "As-Is" and "ToBe" scenarios shows that after applying the model for increasing the accuracy described in point 2.2 , the ranking of four of the five studied alternatives in "To-Be" scenario (A, B, D and E) changes. In the process of selecting a site for establishing an intermodal terminal in the city of Ruse, the leading alternative in both scenarios is alternative $\mathrm{C}$ (Table 8). The rating of the remaining four compared alternatives (A, B, D, and E) changes with improving the accuracy of the estimates (Table 8 ).

The results of the study show that the accuracy of the estimate influences the choice of a leading alternative and can change the ranking of the variants. This is especially true for alternatives with close values of the estimates. As we can see from the results of the research (Table 8), the alternatives that exchange their places (alternatives A and 
$\mathrm{B}$ and alternatives $\mathrm{D}$ and $\mathrm{E}$ ), after improving the accuracy of the estimates, are with close values to those of the estimates for the study conditions. With the "As-Is" scenario the difference in the estimates of the alternatives (Table 2) is significant as compared to the difference in the estimates of the alternatives in the "To-Be" scenario (Table 7). The results of study show that the accumulated inaccuracy through the application of the three-tier scale [3] for evaluating the sub-criteria in selecting a place for construction of an intermodal terminal in the city of Ruse leads to a change in the ranking of the studied alternatives. It is recommended, if possible, to apply more accurate methods for evaluating the alternatives with close values of the multi-criteria estimates.

\section{Conclusion}

The proposed in point 2.2 model for increasing the accuracy of estimates allows us to broaden the scope for applying to the Weighted Sum Model through conversion of the estimates. By converting of dimension values of the criteria into dimensionless grades through the model described in point 2.2, the multi-criterion Weighted Sum Method becomes applicable to a wide range of dimensions. The model could be used for increasing the accuracy of evaluation in selecting a location for establishing an intermodal or other type terminal.

The results of the study show that the model enables comparability of the results obtained for the different options and the application of the model increases the accuracy of the estimates. This can change the final rating of the variants. The model described in the paper is a new approach for application of the Weighted Sum Method. The model could be used for multi-criteria evaluation and comparative analysis among many different alternatives through use the real values of the criteria. The model allows to be used in different types of studies based on the Weighted Sum Method.

\section{References}

1. Integrated Transport Strategy for the period until 2030. Ministry of Transport, Information Technology and Communications, Republic of Bulgaria (2017)

2. Terminology on combined transport. EU, ECMT, UN/ECE (2001)

3. Project "Technical assistance for construction of intermodal terminal in North-Central planning development region in Bulgaria - Ruse" BG161PO004-3.0.01-0004, National Railway Infrastructure Company, Bulgaria (2012)

4. F. Helff, Le Gruenwald, L. d'Orazio, Weighted sum model for multi-objective query optimization for mobile-cloud database environments, CEUR-WS, EDBT/ICDT 2016 Joint Conference, 1558 (2016)

5. E. Mulliner, N. Malys, V. Maliene, Comparative analysis of MCDM methods for the assessment of sustainable housing affordability, Elsevier, Omega, 59(B), March 2016, 146-156, DOI: https://doi.org/10.1016/j.omega.2015.05.013 (2016)
6. E. Triantaphyllou, B. Shu, S. Sanchez, T. Ray, Multicriteria decision making: An operations research approach, Encyclopedia of Electrical and Electronics Engineering, J.G. Webster, Ed., 15, 175-186 (John Wiley \& Sons, New York, 1998)

7. S. Stoilova, An integrated approach for selection of intercity transport schemes on railway networks, Promet - Traffic \& Transportation, 30(4), 367-377, https://doi.org/10.7307/ptt.v30i4.2673 (2018)

8. S. Stoilova, Evaluation efficiency of intermodal transport using multi-criteria analysis. Proceedings of $17^{\text {th }}$ International Scientific Conference Engineering for Rural Development, Jelgava, Latvia, 2030-2039, https://doi.org/10.22616/ERDev2018.17.N246 (2018)

9. A. Deluka-Tibljaš, B. Karleuša, N. Dragičević, Review of multicriteria-analysis methods application in decision making about transport infrastructure, Građevinar 65(7), 619-631 (2013)

10. P. Site, F. Filippi, Weighting methods in multiattribute assessment of transport projects, European Transport Research Review, 1(4), 199-206 (2009)

11. S. Ananiev, S. Martinov, Opportunities of the railway infrastructure in the crossborder region BulgariaRomania for differentiation of freight intermodal centers, Almanac of „Todor Kableshkov” University of Transport, 9, Sofia (2018)

12. P. Jenček, E. Twrdy, Development of regional transport logistics terminal - transport logistics approach, Promet - Traffic \& Transportation, 20(4), 239-249 (2008)

13. G. Odu, O. Charles-Owaba, Review of multi-criteria optimization methods - Theory and applications, IOSR Journal of Engineering (IOSRJEN), 3(10), 01-14 (2013)

14. R. Saaty, The analytic hierarchy process - what it is and how it is used, Mathematical Modelling, 9(3-5), 161-176 (1987)

15. B. Miljković, M. Žižović, A. Petojević, N. Damljanović, New weighted sum model, Filomat, Faculty of Sciences and Mathematics, University of Niš, Serbia, 31(10) 2991-2998 (2017)

16. S. Martinov, Evaluation model of railway infrastructure potential for establishment of freight intermodal terminals, Proceedings of the 5th International Conference on Road and Rail Infrastructures - CETRA 2018, 911-916, DOI: https://doi.org/10.5592/CO/CETRA.2018.907 (2018) 